Forces inside and outside community colleges are changing the context for performance and mandating new conceptions of effectiveness.

\title{
The Future of Institutional Effectiveness
}

\author{
Richard L. Alfred
}

Institutional effectiveness in the fast-paced market that community colleges have operated in for more than two decades is a product of unparalleled expansion. In this market, two basic constructs have served as a basis for measuring effectiveness: a numerator-growth and resources-and a denominator-outputs. Growth in the form of rising enrollments, incremental revenue, and anything else that can be counted is easily calculated and grasped. It is an attractive gauge of effectiveness for leaders looking for quick and easy evidence of success. Outputs—results generated with learners and stakeholders-are not as easily calculated nor are numbers measuring them easily understood. Ironically, while effectiveness models in colleges today focus primarily on outputs, leaders and staff working in a world of enrollments and resources focus on growth. What this suggests is that for leaders, the bigger return on investment may come from working with the numerator rather than measuring the denominator.

The implications of this contradiction for community colleges are many. Chief among them is a disjuncture in how leaders and staff may see effectiveness. Assessment specialists working in the microworld of methodology and numbers should ask themselves how well they understand the context in which effectiveness is measured. If their view of effectiveness includes only the denominator and they do not understand contextual forces influencing the numerator, their contribution to improving performance may be limited. The same is true for leaders working at the interface between institution and environment. If they do not pay attention to the denominator, their capacity to use results to leverage institutional perfor- 
mance may be limited. Simultaneously attending to the numerator and denominator will be important for institutions that are serious about effectiveness.

This chapter addresses the future of institutional effectiveness in community colleges. Its emphasis is on what is measured and why, beginning with a retrospective look at early efforts in effectiveness, moving to an overview of models and methodologies in use today, and closing with a scenario for the future. The contribution of the past can be summed up with the observation that the issue of numerator and denominator has never been resolved. Effectiveness is a complex, multifaceted construct with a myriad of meanings and interpretations. It can be conceptualized and measured in the form of learner outcomes, institutional growth and change, value added, organizational efficiency, stakeholder satisfaction, ratings and rankings, and just about anything else that describes what institutions do. What colleges choose to measure and why is influenced by the context in which they operate. Accordingly, a significant portion of this chapter focuses on contextual conditions facing community colleges and their implications for effectiveness. The chapter closes with a discussion of effectiveness as paradox-an interpretation requiring colleges to simultaneously pursue contradictory approaches to effectiveness to get in front of the change curve.

\section{Effectiveness: A Retrospective Look}

Interest and attentiveness to effectiveness burgeoned in the early 1990s when community colleges entered a period of mission elaboration and enrollment growth. Part and parcel of growth were complexity and a belief that effectiveness was context and situation specific. No conception would prevail over time, nor would any model or measures be universally acceptable to all colleges (Alfred and Kreider, 1991).

The earliest effectiveness models focused on growth and reputation judged relative to other institutions (Roueche and Baker, 1987). As public calls for accountability escalated, new metaphors emerged with indicators of size and reputation no longer sufficient to demonstrate effectiveness. Community colleges were also expected to show evidence of value added through outcomes generated with learners. Different metaphors evolved over time, each adding to the criteria for effectiveness. For example, a metaphor that Alfred and Linder suggested in 1990 focused on the arena in which effectiveness was measured. Some aspects of performance were exclusively generated inside institutional walls (operational efficiency) and were labeled "inside-out," while others evolved outside the institution (employer satisfaction) and were labeled "outside-in." The California Association of Community Colleges developed a functional model of effectiveness in which indicators describing the performance of specific operating units or "functions" (educational programs, student services, academic support services, and so forth) served as a focal point for assessment 
(Doucette and Hughes, 1990). Midlands Technical College in South Carolina developed and implemented a critical success factors approach to effectiveness in which assessment was focused on performance characteristics important to the success of the college and the expectations of its stakeholders (Hudgins, 1990).

These early approaches led to the development of the first edition of Core Indicators of Effectiveness for Community Colleges in 1994, a report written by a roundtable of community college educators representing different streams of work on effectiveness (Community College Roundtable, 1994). The goal of Core Indicators was to help community college practitioners respond coherently to a simple but important question: What are the key indicators of effectiveness in community colleges? The work of the roundtable focused primarily on student outcomes and stakeholder satisfaction as indexes of effectiveness and provided a working definition and technical descriptions for thirteen indicators. This volume was followed by a second edition of Core Indicators in 1999 that incorporated information about changing contextual conditions for effectiveness and expanded the number of indicators to fourteen by adding licensure and certification pass rates (Alfred, Ewell, Hudgins, and McClenney, 1999). New in this edition was cautionary advice regarding "red lights" that colleges needed to be aware of in effectiveness assessment and suggestions for responding to externally imposed measures. The third edition of Core Indicators, published in 2007, advanced the number of core indicators to sixteen by adding measures of student learning and general education competencies and presented a stage model that colleges with varying capability for assessment could use to measure effectiveness (Alfred, Shults, and Seybert, 2007).

The Core Indicators editions did much to advance our understanding of effectiveness, but they were limited by a linear conception of effectiveness. Their focus was exclusively on the denominator: outputs. They failed to account for the numerator-growth and resources-and contextual conditions shaping it. Moreover, they were written in the context of existing forces and conditions. The future was not the subject of speculation nor were ways of envisioning effectiveness based on intangibles such as stakeholder perceptions, faculty and staff satisfaction, and leveraging.

\section{Change and Contradiction}

Before the recession beginning in 2008, the future for community colleges was challenging but at least understandable. At the beginning of 2011, it is an uncharted horizon of simultaneously contradictory forces of growth and opportunity, resource decline, intensifying pressure for accountability, and changing rules of competition. Every college will encounter opportunities for growth and development that are part of a market loaded with customers wanting more and competitors offering more. Counterbalancing these opportunities, however, will be uncertainty about the resources that 
community colleges will have to support growth and their capacity to absorb growing legions of learners. These forces will have a significant impact on how colleges conceptualize and measure effectiveness.

Forces of Change. Substantive change in the terrain for effectiveness can be traced back to 2008 with the onset of the recession and the election of Barack Obama as president. The recession profoundly disrupted every aspect of American life. Mobility ground to a sixty-year low as unemployment, plunging home values, and declining confidence in the economy forced people to delay major life decisions. The employment decline between October 2007 and April 2010 was the steepest on record since 1945 (U.S. Bureau of Labor Statistics, 2010). Millions of Americans became prisoners of their circumstances as the net worth of households dropped 22 percent from its peak in June 2007 (Federal Reserve Bank, 2010). A survey of 2,002 adults in April 2010 by Citi indicated that Americans, by more than a two-to-one margin, believed that they were worse off financially than they were the prior year (Clements, 2011). Some were unguardedly pessimistic about future prospects: for example, 36 percent of the adults surveyed believed the economy had hit bottom, but 59 percent believed economic conditions had not yet bottomed out. The mood of public officials paralleled the plight of citizens as governors in all but a few states indicated that state economies had hit bottom but were not yet in recovery, more federal government expenditures would be needed to create jobs and spur economic growth, and without renewal, exhausted stimulus funds would lead to further deterioration of public college and university operating budgets in fiscal years 2011 and 2012.

But in every cloud there is a silver lining. For community colleges, it has come in the form of rising visibility and dramatic enrollment growth. Enrollment increased by 10 to 20 percent between 2008 and 2009, and demand is expected to rise as community colleges become institutions of choice for displaced workers and families seeking relief from rising educational costs (Alfred, 2009). Growth, however, is not always positive. It has a downside realized in imbalance between demand and capacity when resources are insufficient to support growth. Colleges experiencing simultaneous forces of record enrollment growth and falling revenues have reached a breaking point in capacity. The instructional day has been extended, more part-time faculty have been hired, class size has been increased, and space has been acquired whenever and wherever possible. The large-scale addition of temporary staff has increased organizational size and complexity and further fragmented culture as new silos have emerged and existing silos have been reinforced. The dramatic increase in the rate of growth has prompted concerns about quality in institutions in which temporary staff deliver a significant portion of their core process.

With enrollment growth and problems with capacity have come policy initiatives that simultaneously favor community colleges and demand more of them. In 2009, the Obama administration launched a $\$ 12$ billion com- 
pletion initiative to boost the number of college graduates by 5 million by 2020. Community colleges will be on the receiving end of a sizable portion of this money, but strings are attached. They will be expected to value completion as much as access and to improve graduation rates significantly. This amounts to a dynamic of contradiction: community colleges will grow, but they will have less to work with and more will be expected of them.

The Dynamic of Contradiction. Contrary forces of growth and decline could be likened to decelerators and accelerators. As decelerators, they constrain movement by constricting resources available to colleges, and as accelerators, they facilitate movement by encouraging change. Although community colleges are working with less, learners do not diminish their expectations and wait for them to catch up. They continually raise the bar on what they want and expect, and colleges must find ways to deliver or face consequences. In effect, decelerators become accelerators when leaders and staff must find creative solutions to adversity.

Accelerators and decelerators make up a dynamic of contradiction that will shape the context for effectiveness in the future. On the one hand, if forces of deceleration-a slowly recovering economy, declining public support, and diminished capacity-maintain their grip, enrollment could plateau as institutional capacity falls short of demand. If colleges choose to deliver more of their core process through temporary staff, nagging questions about quality and accountability could emerge. Stakeholders believing they are getting less will invariably push for more, with the result that leaders may be forced to divert resources from access to performance. Institutions focusing on growth and failing to attend to outputs will do so at their own peril in a policy landscape requiring incremental evidence of accountability.

If the economy instead moves through a sustained recovery, a scenario driven purely by forces of deceleration is unlikely. During a recovery, people return to a pattern of consumption, resulting in increased spending and an influx of revenue into state treasuries that eventually finds its way into community college operating budgets and fuels new growth. For community colleges, the implication of simultaneous conditions of growth and reduction would be one of paradox. While coping with the effects of deceleration fueled by lingering recession, they will simultaneously be coping with forces of acceleration fueled by calls for growth and accountability. Learners will want more and better service, and policymakers will push for evidence of better results. The implication for community colleges will be a future in which multiple approaches to the conception and measurement of effectiveness are employed to address contradictory conditions.

\section{Paradox of the Future}

Effectiveness is likely to operate in two realms in the future-one that can be called objective and the other subjective. These realms are contradictory 
in nature and contribute to a condition of paradox that will mark the future of effectiveness in community colleges.

Objective Realm. The term objective refers to events in the realm of experience that are independent of individual thought and readily perceptible to all observers. Indicators consensually recognized as fundamental to the mission of community colleges, deeply embedded in external mandates, and of high importance to important stakeholders can be said to comprise an objective basis for effectiveness. Indeed, the indicators making up this realm are integral to the effectiveness models in most colleges:

- Rate of growth (enrollment, programs, budgets, staff, facilities, and so forth)

- Efficiency (use of resources)

- Persistence

- Graduation rate

- Success in subsequent related course work

- Program learning outcomes and mastery of discipline

- General education competencies

- Career program placement rates

- Licensure and certification pass rates

- Client satisfaction with programs and services

- Transfer rate

- Performance after transfer

The basis for effectiveness models in the objective realm is the ability of a college to produce outputs that correspond to statements of purpose in its mission and vision (Ewell, 1992) and the needs and expectations of its stakeholders (Alfred, 2005). This implies that the mission of a college and the outcomes it generates are consistent with a growing variety of stakeholder needs. Nothing is more transparent to stakeholders than simply reported numbers measuring the "objective" face of effectiveness-particularly measures related to end goals like degree completion, transfer, and job attainment and advancement. Because the numbers that make up this realm are central to what community colleges do and what is expected of them, it is reasonable to expect that they will be part of the effectiveness equation in the foreseeable future.

Subjective Realm. We live in a world in which intangibles are becoming increasingly important. Policymakers and practitioners alike point to the growing importance of values, ideas, beliefs, and perceptions in understanding performance in organizations. Whether a college performs well or poorly is as much a function of stakeholder perceptions of the outcomes it generates as the outcomes themselves. In this way, effectiveness has a subjective dimension realized in how audiences interpret the numbers generated in a college's objective realm.

The subjective realm of effectiveness, new to most colleges, will receive increasing attention in the future. It will take at least three forms on most 
campuses: valuation, in which effectiveness is a measure of the feelings people hold toward an institution; stretch, in which effectiveness is a measure of an institution's capacity to leverage its resources; and interpretation, in which effectiveness is a measure of a college's ability to create (and induce stakeholders to accept) new and different conceptions of success.

Effectiveness as Valuation. Valuation-the process through which stakeholders form perceptions of colleges by the results they generate and report-will become as important a contributor to effectiveness as the results themselves. Effectiveness is not absolute: it is linked to feelings and beliefs, and it is a product of the experience people have with institutions. Most of today's effectiveness models were created when linear relationships prevailed between institutions and stakeholders. Tomorrow's models will be sensitive to the fact that the influence of stakeholders has grown, and more information is needed from them to gauge a college's performance. Effectiveness will become a correlate of the relationship between stakeholder needs and satisfaction. Factors that change or alter this relationship will need to be identified and measured. For example, as competitors and technology change the rules of customer service, the connection between what stakeholders want (more and better service) and satisfaction (experience that meets or exceeds expectations) will need to be reexamined. Conventional indicators will have some utility in measuring this connection, but they may not dig deeply enough into the minds of individual stakeholders to uncover deeply seated feelings and beliefs.

We can illustrate the importance of valuation in effectiveness using a customer service analogy. If currently enrolled students indicate they are satisfied with college support services on routine surveys but still transfer or drop out what is really being measured? Conventional effectiveness models would leapfrog the process indicators involved in satisfaction and focus on outcomes-one of which would be nonpersistence. These models are not designed to measure student satisfaction as a function of the difference between what students want and expect and what they are getting. Future-focused effectiveness models will pay more attention to deeply embedded feelings students develop and hold toward the institution. In contrast to close-ended surveys that elicit surface impressions, these models will employ methods of data gathering that probe beneath the surface of what students are thinking and find out how they really feel. Factors typically not included in surveys, such as the nature and variety of contacts with faculty and staff, difficulty in accessing and using services, and feelings of social and cultural isolation, will be probed and their effect on satisfaction determined.

What valuation will bring to effectiveness in the future is the realization that community colleges will need to find new ways of measuring things that really count. They will learn not to rely solely on outcome 
measures and conventional techniques for gathering information. They will create breakthroughs in information depth and substance by incorporating measures of process into effectiveness models and using informationgathering techniques that uncover stakeholders' deepest concerns.

Effectiveness as Stretch. When colleges rely primarily on indicators of output to measure effectiveness, attention goes to indexes that gauge the bottom line: the rate of growth, learner outcomes, placement rates, and the like. Institutions facing tough market conditions and diminishing resources experience a different reality that affects their approach to effectiveness. Intangibles underlying performance like organizational culture, employee satisfaction, and motivation have as much to do with outcomes. It will not be enough for leaders to run numbers related to growth and learner outcomes to establish that a college is doing well or poorly. They will also need to run numbers that gauge a college's ability to leverage its resources and, in so doing, enhance its capacity. This is called stretch, and it refers to an institution's capacity to achieve superior performance-that is, to maximize effectiveness-by optimizing its resources (Alfred, Shults, Jaquette, and Strickland, 2009).

How would stretch work in the effectiveness equation for community colleges? An obvious problem for colleges experiencing sustained erosion of resources will be locating new sources of revenue to support growth and maintain performance. By focusing explicitly on intangible resources such as people, ideas, culture, and tacit knowledge, stretch will encourage institutions and leaders to broaden their view of effectiveness to include often overlooked human dynamics that are important to performance (Alfred, Shults, Jaquette, and Strickland, 2009). Feldman and Khademian (2003) provide a good illustration in their examination of empowerment and cascading vitality in organizations resulting from the virtuous behavior of leaders. Actions that leaders take to empower staff work to improve relationships between individuals, organizations, and communities; increase engagement; and result in improved performance. Employee engagement leads to increased meaningfulness in work through its effect on job design, which in turn results in more motivated employees. In other words, positive actions or events at the individual level (empowerment by leaders) can lead to favorable outcomes at the organizational level (improved performance through employee engagement) that can create positive outcomes for the individual (meaningfulness).

Conceptualization and measurement of stretch could become an important dimension of effectiveness for community colleges because of its amplifying effect on resources. It does not develop without internal cohesion, so cohesion will become a goal within many colleges and efforts will be made to define and measure it. End measures (outcomes) will be seen as insufficient in and of themselves for assessing effectiveness. Effective colleges will be seen as those that emphasize, and are able to document, stretch in addition to outcomes. 
Effectiveness as Interpretation. Since their inception, community colleges have been subjected to traditional definitions of completion and student success by external agencies. As a result, the focus of effectiveness models has narrowed over time to tested measures of outcomes, and creative thinking has atrophied. Now, however, community colleges are under significant pressure to focus on completion and to push more students across the finish line. The usual agents of accountability-government agencies, accrediting associations, and foundations-are clear about their expectations for completion and show no signs of backing off.

As institutions and leaders cope with increased pressure for completion, they will come to understand that it will not be enough to "do better" on traditional measures of success-to incrementally increase the number of graduates or completers. Instead they will need to establish new criteria for success by reinterpreting what completion means while simultaneously improving performance on established measures. To accomplish this, they will need to tap into the creative instincts of faculty and staff to frame new conceptions of "finished products," new completion structures, and new ways of measuring completion. They will begin this process by asking five questions of themselves:

- What is the reality of completion for students enrolled in this institution? In most institutions, a predictable pattern of results forms with entering student cohorts. Norms for completion are established as the proportion of learners graduating, transferring prior to graduation, dropping out, and so on remains constant from year to year. This pattern says a lot about an institution's potential for creating finished products. Strategically thinking leaders will use it to gauge the need within their institutions for new conceptions of completion.

- Do we assign equal importance to completion and access? Colleges that make meaningful inroads into student completion begin by underscoring its importance and assigning it equal value with access. They understand, however, that mandates do not guarantee commitment. They program in intangibles in the form of leaders and staff who can transform a belief in the importance of completion into the reality of achievement. Even the most compelling belief will lose its power if it floats unconnected above the everyday reality of organizational life. Identifying people and devising processes to bridge commitment to completion into the everyday work of staff is a role that leaders play in colleges with a capacity to innovate.

- Are the hydraulics of our college-its mission, policies, culture, organizational architecture, systems and processes, and operations-designed to encourage high levels of completion? Hydraulics are the mechanisms by which institutions translate mission, objectives, and resources into aligned action by individuals throughout the organization. In many colleges, attention to completion lags when the organization is overloaded 
with multiple and conflicting priorities. Leaders interested in optimizing completion pay careful attention to the relationship between hydraulics and institutional goals and take purposeful steps to ensure convergence.

- Do we have the imagination and creativity to envision new forms of completion and devise practical plans for implementation? In colleges willing to experiment with new conceptions of completion, investment in ideas is as important as problem solving and operations. Leaders in these colleges encourage staff to engage in conversations that open the door to innovation. These conversations focus on questions that cut to the core of issues surrounding completion: What will "completion" mean in the future, and how will it differ from what it means today? What forms must completion take to meet the changing needs of students and external stakeholders? How do we need to change the institution to embrace new forms of completion? How can we commit people to new and different forms of completion?

- Do we have the resources and wherewithal to convince external audiences to accept alternative conceptions of completion? Colleges experimenting with completion will be lauded for their efforts, but effort will be inconsequential unless they are able to convince funding agencies and policymakers to accept new conceptions of completion. This will be a tough sell because of ingrained attitudes and policy implications, but it is essential if community colleges are to level the playing field for completion.

By forging new conceptions of completion and legitimizing them with external audiences, community colleges will essentially broaden the framework for effectiveness. New outcomes will be factored into effectiveness models including credentials, course packages leading to meaningful employment, life experience, and myriad curricular and cocurricular experiences that more fully and accurately depict what completion means in community colleges.

\section{Conclusion}

New applications of effectiveness will evolve as contextual conditions change and require community colleges to do more and better with lessmore service for more learners and better outcomes with fewer resources. The arena for effectiveness will expand to include intangibles that have an impact on institutional performance. Prominent in this arena will be measures of capacity and leverage that gauge how effectively institutions use resources. Implicit in all of this will be an urgency for action. Community colleges will need to adjust effectiveness models in accord with changing contextual conditions and craft interpretations of performance that fit the reality of the landscape, their resources, and their capacity. 


\section{References}

Alfred, R. L. Managing the Big Picture in College and Universities: From Tactics to Strategy. New York: Praeger, 2005.

Alfred, R. L. "Navigating Change with a Conventional Organization." New Jersey Association of Community College Presidents, speech, Nov. 19, 2009.

Alfred, R. L., Ewell, P., Hudgins, J., and McClenney, K. Core Indicators of Effectiveness for Community Colleges. (2nd ed.) Washington, D.C.: Community College Press, 1999.

Alfred, R. L., and Kreider, P. "Creating a Culture of Institutional Effectiveness." Community College Journal, Apr.-May 1991, pp. 34-39.

Alfred, R. L., and Linder, V. Rhetoric to Reality: Effectiveness in Community Colleges. Ann Arbor, Mich.: Community College Consortium, 1990.

Alfred, R. L., Shults, C., Jaquette, O., and Strickland, S. Community Colleges on the Horizon: Challenge, Choice, or Abundance. Lanham, Md.: Rowman and Littlefield, 2009.

Alfred, R. L., Shults, C., and Seybert, J. Core Indicators of Effectiveness for Community Colleges. (3rd ed.) Washington, D.C.: Community College Press, 2007.

Clements, J. "New Citi Survey: Americans Are Mired in Economic Winter Despite Signs of Spring." Retrieved from http://new.citi.com/2010/04/new-citi-survey-americans-are -mired-in-economic-winter-despite-signs-of-spring shtml on February 20, 2011.

Community College Roundtable. Community Colleges: Core Indicators of Effectiveness. Washington, D.C.: American Association of Community Colleges, 1994.

Doucette, D., and Hughes, B. (eds.). Assessing Institutional Effectiveness in Community Colleges. Laguna Hills, Calif.: League of Innovation, 1990.

Ewell, P. T. Outcomes Assessment, Institutional Effectiveness, and Accreditation: A Conceptual Exploration. Resource Paper for the Council on Postsecondary Accreditation Task Force on Institutional Effectiveness, 1992.

Federal Reserve Bank. 24 Flow of Funds Report. Washington, D.C.: Federal Reserve Bank, Mar. 11, 2010.

Feldman, M. S., and Khademian, A. "Empowerment and Cascading Vitality." In K. S. Cameron, J. E. Dutton, and R. E. Quinn (Eds.), Positive Organizational Scholarship: Foundations of a New Discipline. San Francisco: Berrett-Koehler.

Hudgins, J. Institutional Effectiveness Report Card. Columbia, S.C.: Midlands Technical College, 1990.

Roueche, J., and Baker, G. Access and Excellence: The Open-Door College. Washington, D.C.: Community College Press, 1987.

U.S. Bureau of Labor Statistics. "Payroll Employment in 2009: Job Losses Continue." Monthly Labor Review. Washington, D.C.: Department of Labor, 2010.

RICHARD L. ALFRED is professor of higher education at the University of Michigan and founding director of the Center for Community College Development. 